\title{
Sky brightness at the ESO La Silla Observatory 1978 to $1988^{\star, \star \star}$
}

\author{
K. Mattila ${ }^{1}$, P. Väisänen ${ }^{1}$ and G.F.O. v. Appen-Schnur ${ }^{2}$ \\ 1 Observatory, P.O. Box 14, FIN-00014 University of Helsinki, Finland \\ 2 Astronomisches Institut, Ruhr-Universität Bochum, D-44780 Bochum, Germany
}

Received November 4, 1995; accepted January 13, 1996

\begin{abstract}
We have measured the sky brightness at the European Southern Observatory (ESO), La Silla, during 40 moonless nights in the years 1978 - 1988. Most of the measurements were performed in five intermediate band filters between 3500 and $5470 \AA$, including the Strömgren $u, b$, and $y$ bands. In addition we made measurements in the uvgr and $B V R I$ photometric systems. Typically 20 to 40 readings were obtained each night at a fixed $(\alpha, \delta)$ position, selected to be free of stars down to a limiting magnitude of $B \approx 20$. The sky brightnesses were calibrated using standard stars. We discuss the sky brightness variations both during individual nights and from year to year. We have found that the long-term variations are well correlated with the solar activity, for which the $10.7 \mathrm{~cm}$ radio flux is used as a measure. We compare the sky brightnesses as observed at La Silla with the published values for several other sites. Finally, based on the tight correlation of the airglow variations in two pass bands near $4000 \AA$ we propose a new method for the separation of airglow and zodiacal light contributions.
\end{abstract}

Key words: atmospheric effects — site testing — techniques: photometric — interplanetary medium

\section{Introduction}

The brightness of the night sky at an undisturbed site consists of several natural components of both extraterrestrial and atmospheric origin, including the extragalactic background light (EBL), the integrated light of stars (ISL), the diffuse galactic light (DGL), the zodiacal light (ZL), the airglow (AGL) and aurora, as well as the scattered light from the earth's troposphere. During the past decades man-made lighting has caused, via its scattering in the troposphere, an ever-increasing additional contribution to the light of the night sky (LONS). This light pollution has been of great concern to the astronomical community (see e.g. McNally 1994) and raises the need, besides measures taken to protect the sky, also for more observing campaigns. There has also been interest from geophysicists to have new night sky data for comparison with the airglow monitoring conducted during the International Geophysical Year in 1957-58.

Besides its absolute level another important aspect of the sky brightness is its long- and short-term variability

\footnotetext{
Send offprint requests to: K. Mattila

* Based on observations made at the European Southern Observatory, La Silla, Chile

${ }^{\star \star}$ Figs. 3 and 4 only available in electronic form at CDS via ftp 130.79.128.5
}

which is caused by the varying level of airglow, and, to a lesser extent, by the varying tropospheric conditions. While the short-term variability (minutes to hours) may be harmful for observations of very faint sources the longterm variability $(\sim$ solar cycle) may mask the increasing level of light pollution.

Long-term observing campaigns have been carried out at several observatories and potential sites to determine the total natural sky background as well as to monitor the level of sky pollution by artificial lighting (see e.g. Lyutyi \& Sharov 1982; Walker 1988; Pilachowski et al. 1989; Krisciunas 1990; Leinert et al. 1995). For the ESO observatory at La Silla very few data on the sky brightness have been published so far. The $B V R I$ values given in The ESO Users Manual (Schwarz \& Melnick 1989) refer to earlier, rather scanty observations by the present authors (Schnur \& Mattila 1979). La Silla is one of the very few major observatory sites with no or only negligible pollution from artificial lighting. The nearest cities are Vallenar, $65 \mathrm{~km}$ NNW, and La Serena/Coquimbo, $150 \mathrm{~km}$ SW of La Silla, with populations of 38000 and 164000, respectively. Their combined contribution to the sky brightness at La Silla can be estimated to be less than 0.02 mag (Walker 1977).

In the present paper we report on an extensive series of sky brightness measurements at La Silla during the years 
1978-1988. The sky brightness data were obtained as a byproduct of long-term projects aimed at the measurement of the extragalactic background light and the diffuse light in clusters of galaxies. The observational procedures and reduction techniques are described in Sect. 2. The results are presented in Sect. 3. In Sect. 4 we discuss the sky brightness variations and their correlation with solar activity. In Sect. 5 we compare the sky brightness values as measured at La Silla with other observing sites. In Sect. 6 we suggest a new method for the separation of airglow and zodiacal light, and in Sect. 7 we present our summary and conclusions.

\section{Observations and reductions}

The observations were carried out at the European Southern Observatory, La Silla, Chile. The observatory is located at $\lambda=70^{\circ} 43.8^{\prime} \mathrm{W}, \varphi=-29^{\circ} 15.4^{\prime}$ and at a height of $2350 \mathrm{~m}$. Two telescopes were used simultaneously, the ESO $1-\mathrm{m}$ and 50-cm telescopes, located at a distance of ca. 200 meters from each other. Each telescope was equipped with a standard single-channel photoelectrical photometer.

\subsection{Intermediate band pass data}

Our intermediate band observations were carried out primarily for the purpose of separating the extragalactic background light from the other night sky brightness components. The method utilizes the shadowing effect of a high-galactic latitude dark nebula (cf. Mattila et al. 1991). A two-telescope measuring technique was introduced for the purpose of eliminating the airglow variations. The 50$\mathrm{cm}$ telescope was used as a monitor and was pointed towards the same position on the sky $(\alpha, \delta$ fixed) in or near the dark cloud during the whole measuring cycle. The 1$\mathrm{m}$ telescope was used to measure several individual starfree positions within and outside the dark cloud in a total area covering some 5 degrees on the sky. A "standard" position was measured typically from one to three times each hour at the 1-m telescope. The 50-cm telescope data could have been used for the present purpose also alone, without the 1-m telescope data. However, the calibration was done with the 1-m telescope only. Also, because of the much larger focal plane aperture size used at the 50cm telescope the contribution of starlight was unavoidably present whereas it was excluded down to the limiting magnitude of $B \approx 20 \mathrm{mag}$ in the $1-\mathrm{m}$ data.

For these reasons, only the "standard" position values from the 1-m data were used to get the final sky brightness directly. However, the 50-cm data were used, in addition, to get "effective" standard position measurements; this could be done because the ratio of the responses from the two telescopes remains very stable during the night despite the temporal variations of the airglow. For the standard positions we calculate the ratio of the $50-\mathrm{cm}$ and $1-\mathrm{m}$ counts and then interpolate the ratio values corresponding to measurements between two consequtive standard positions. A simple linear interpolation is justified by the stability of the ratio values. Dividing the value of $50-\mathrm{cm}$ in-between -count with the interpolated ratio, we get the corresponding count rate for the 1-m standard position. This method was used in connection with the reductions of all the intermediate band data.

Our observing method, although not specifically intended for the total night sky brightness measurements has some advantages as compared with the previous night sky brightness measurements: (1) we have selected fixed, well-identified positions which were repeatedly observed in the different nights and years; thus, the differences (in zodiacal light and galactic light components) between different $\alpha, \delta$ positions on the sky do not introduce scatter in our data; $(2)$ the positions are selected to be free of stars down to $B \approx 20$ mag. Thus, the starlight contribution is negligible and does not introduce additional scatter. The elimination of the starlight component is especially important when measuring in the "pseudocontinuum" of the airglow spectrum; (3) for multicolour observations it is important that all colours are are observed at the same position and at nearly the same time; (4) because the zodiacal light contribution varies greatly over the sky it is important that the measured positions are known and the ZL differences are taken into account when comparing different data sets.

The sky brightness measurements in the intermediate band filters were carried out in a total of 24 nights in four sets of observations: June 22-24, 1979, December 6-10, 1980, December 15-20, 1987, and December 2-11, 1988. The dark cloud L134 was observed in the year 1979 and L1642 in the rest. The coordinates for the "standard" observing positions are given in Table 1 . The airmass values as function of the local sidereal time are shown in Fig. 1.

The diameter of the focal plane aperture used at the 1-m telescope was $6.40 \mathrm{~mm}$ corresponding to 86.55 arc sec on the sky. The effective aperture solid angle was determined by comparing the sky signal of this larger (non-uniform) aperture with the sky signal measured with smaller apertures (assumed to be uniform). It was found to be $4.1310^{-4}$ sq. degrees or $1.2710^{-7}$ sterad, corresponding to a "beam efficiency" of 0.91 . (This beam efficiency is wrt the response at the centre of the apertures where the standard stars were placed during the calibration measurements.) At the 50-cm telescope, in order to maximize the signal, no focal-plane aperture was used. This resulted in a non-uniform beam corresponding to an effective aperture diameter of $\sim 7^{\prime}$.

The photomultipliers were of the same type at both telescopes: EMI 6256S in 1979 and 1980 and EMI 9789 QB in 1987 and 1988. In the years 1980 and 1987 we used five filters, $u(3500 \AA), 3840 \AA(\Delta \lambda=175 \AA), 4160 \AA(\Delta \lambda=$ $140 \AA), b(4670 \AA)$, and $y(5470 \AA)$. Of these, $u, b$ and $y$ 
Table 1. Coordinates of the observed positions and an estimate for the intensity of the zodiacal light in units of $S_{10}(\mathrm{G} 2 \mathrm{~V})$

\begin{tabular}{|c|c|c|c|c|}
\hline Position & $\alpha_{1950}$ & $\delta_{1950}$ & $\bar{\beta}$ & $I_{\mathrm{ZL}}$ \\
\hline CL004.8-3450 & $0 \mathrm{~h} 02 \mathrm{~m} 56.4 \mathrm{~s}$ & $-35^{\circ} 10^{\prime} 54^{\prime \prime}$ & -32.2 & 85 \\
\hline S245 & $3 \mathrm{~h} 59 \mathrm{~m} 03.8 \mathrm{~s}$ & $2^{\circ} 08^{\prime} 49^{\prime \prime}$ & -18.0 & 124 \\
\hline L1642 Pos. 8 & $4 \mathrm{~h} 32 \mathrm{~m} 53.4 \mathrm{~s}$ & $-14^{\circ} 22^{\prime} 33^{\prime \prime}$ & -35.9 & 94 \\
\hline L1642 Pos. 7 & $4 \mathrm{~h} 33 \mathrm{~m} 18.0 \mathrm{~s}$ & $-14^{\circ} 21^{\prime} 27^{\prime \prime}$ & -35.9 & 94 \\
\hline A 496 & $4 \mathrm{~h} 31 \mathrm{~m} 18.0 \mathrm{~s}$ & $-13^{\circ} 21^{\prime} 00^{\prime \prime}$ & -34.8 & 87 \\
\hline A 754 & $9 \mathrm{~h} 04 \mathrm{~m} 40.5 \mathrm{~s}$ & $-9^{\circ} 19^{\prime} 58^{\prime \prime}$ & -24.9 & 112 \\
\hline A1060 & $10 \mathrm{~h} 34 \mathrm{~m} \mathrm{30.0s}$ & $-27^{\circ} 16^{\prime} 00^{\prime \prime}$ & -33.3 & 87 \\
\hline A1146 & $10 \mathrm{~h} 58 \mathrm{~m} 54.0 \mathrm{~s}$ & $-22^{\circ} 27^{\prime} 00^{\prime \prime}$ & -28.6 & 90 \\
\hline L134 & $15 \mathrm{~h} 50 \mathrm{~m} 50.6 \mathrm{~s}$ & $-4^{\circ} 25^{\prime} 28^{\prime \prime}$ & 15.3 & 115 \\
\hline
\end{tabular}

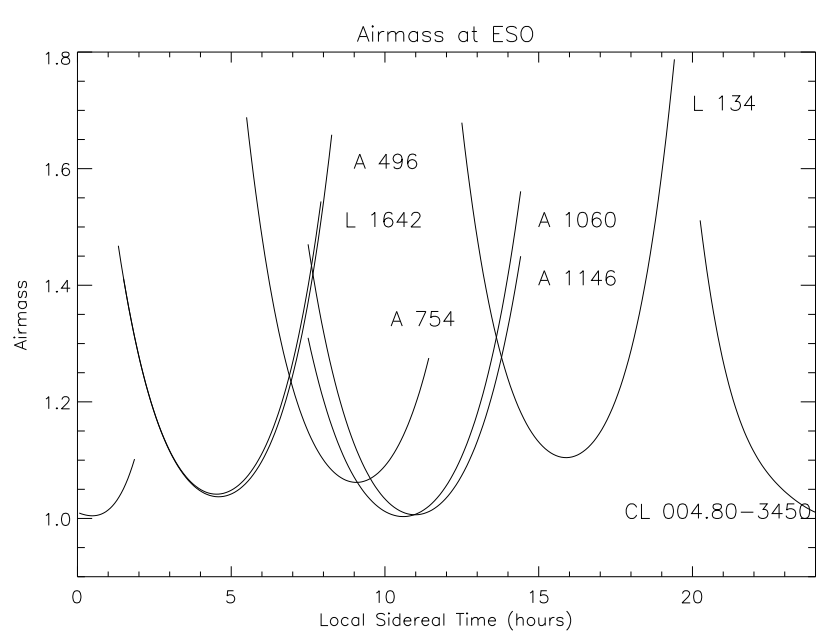

Fig. 1. Airmass as a function of local sidereal time for the targets. The LST intervals indicate the ranges for our measurements

refer to the standard Strömgren system and the other two are interference filters manufactured by Omega Optical Inc. (halfwidths in parentheses). In the year 1979 the $u$ filter was not in use; in the year 1988 seven intermediate band filters were used; in addition to the previous ones we had interference filters centered at $5010 \AA$ ([OIII]; $\Delta \lambda=$ $20 \AA)$ and $4870 \AA\left(\mathrm{H}_{\beta}\right.$ narrow; $\left.27 \AA\right)$. The response curves of all the filters are given in Fig. 2. The observations were carried out as varying sequences from year to year, e.g. $u, b, y, 3840,4160, u$, in the year 1987 , with a 30 to $60 \mathrm{sec}$ integration time per filter.

The sky brightness values, $f_{\lambda}$, for the intermediate band data are expressed in units of $10^{-9} \mathrm{ergs}^{-2} \mathrm{~s}^{-1}$ sterad $^{-1} \AA^{-1}$. The calibration and the determination of extinction coefficents were done by observing every night ca. 10 spectrophotometric standard stars from the lists of Stone (1977) and Oke (1974). A journal of observations and the extinction coefficients are given in Table $2 \mathrm{a}$. We should emphasize that our sky brightness values are as actually observed from the ground with no extinction correction made. An extinction correction would not be

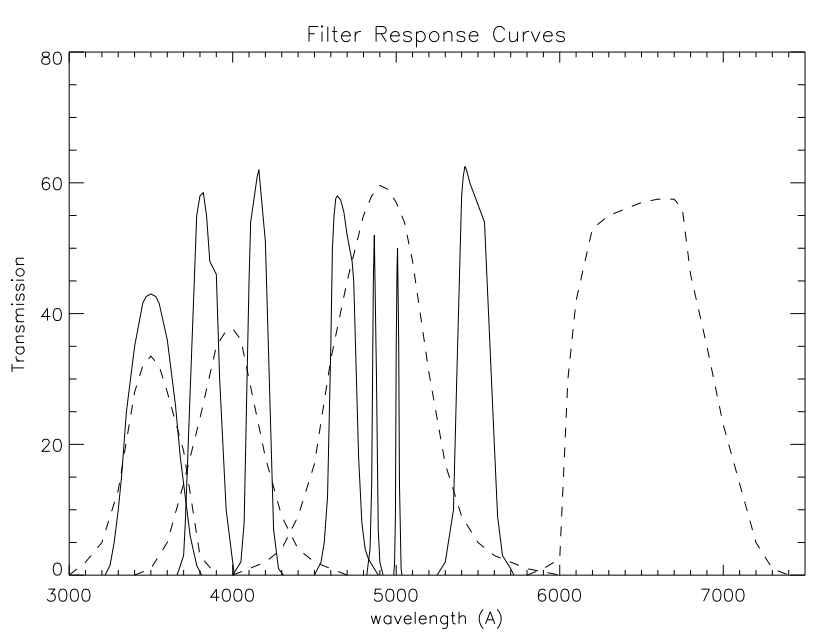

Fig. 2. Spectral responses of the filters. The intermediate band filters are drawn with full line, the uvgr filters with dashed line

meaningful for the present purpose. The extinction coefficients are needed, however, for the determination of the calibration factors in terms of (extraterrestrial) stellar fluxes.

\subsection{BVRI data}

The $B V R I$ broad band sky brightness measurements were carried out in the four consequtive nights February 58 1978. The primary aim of these measurements was to determine the diffuse light in galaxy clusters. The broadband sky brightnesses to be reported here were obtained and calibrated at the 1-m telescope alone. The standard set of $B, V, R$, and $I$ filters available at ESO, La Silla was used. The aperture size was 60.86 arcsec and was assumed to have a "beam efficiency" of 1 . The type of photomultiplier was RCA 31034A. The minimum count rate for the sky readings was $\sim 3000$ counts/s.

The sky brightnesses were calibrated and atmospheric extinction coefficients were determined by observing in each night ca. 10 standard stars $(V=10-13 \mathrm{mag})$. A journal of observations and the extinction coefficients are 
Table 2. Journal of observations and extinctions coefficents

a) Intermediate band observations

\begin{tabular}{|c|c|c|c|c|c|c|c|c|c|}
\hline \multirow[t]{2}{*}{ Date } & \multirow{2}{*}{$\begin{array}{l}\text { Targets } \\
\text { observed }\end{array}$} & \multicolumn{7}{|c|}{ Extinction coefficents } & \multirow[t]{2}{*}{ Remarks } \\
\hline & & $u$ & 3840 & 4160 & $b$ & $y$ & OIII & $\mathrm{H} \beta$ & \\
\hline $1979-06-22$ & L 134 & - & 0.41 & 0.33 & 0.22 & 0.17 & - & - & \\
\hline 23 & L 134 & - & 0.41 & 0.33 & 0.22 & 0.17 & - & - & \\
\hline 24 & L 134 & - & 0.41 & 0.33 & 0.22 & 0.17 & - & - & \\
\hline $1980-12-06$ & L 1642 & 0.66 & 0.42 & 0.32 & 0.20 & 0.14 & - & - & \\
\hline 07 & L 1642 & 0.82 & 0.44 & 0.35 & 0.22 & 0.18 & - & - & \\
\hline 08 & L 1642 & 0.64 & 0.43 & 0.33 & 0.22 & 0.15 & - & - & \\
\hline 09 & L 1642 & 0.57 & 0.39 & 0.28 & 0.17 & 0.11 & - & - & \\
\hline 10 & L 1642 & 0.59 & 0.40 & 0.30 & 0.19 & 0.11 & - & - & \\
\hline $1987-12-15$ & L 1642 & 0.61 & 0.37 & 0.29 & 0.20 & 0.13 & - & - & \\
\hline 16 & L 1642 & 0.51 & 0.30 & 0.26 & 0.16 & 0.12 & - & - & \\
\hline 17 & L 1642 & 0.53 & 0.36 & 0.25 & 0.16 & 0.11 & - & - & \\
\hline 18 & L 1642 & 0.54 & 0.35 & 0.27 & 0.15 & 0.12 & - & - & \\
\hline 19 & L 1642 & 0.59 & 0.35 & 0.26 & 0.17 & 0.12 & - & - & 1 \\
\hline 20 & L 1642 & 0.56 & 0.34 & 0.25 & 0.14 & 0.11 & - & - & \\
\hline $1988-12-02$ & L 1642 & 0.50 & 0.34 & 0.23 & 0.14 & 0.11 & - & 0.12 & 2 \\
\hline 03 & L 1642 & 0.50 & 0.34 & 0.23 & 0.14 & 0.11 & - & 0.12 & 2 \\
\hline 04 & L 1642 & 0.52 & 0.39 & 0.24 & 0.14 & 0.11 & 0.15 & 0.15 & \\
\hline 05 & S 245 & 0.50 & 0.34 & 0.23 & 0.14 & 0.11 & - & 0.12 & 2 \\
\hline 06 & S 245, L 1642 & 0.50 & 0.34 & 0.23 & 0.14 & 0.11 & - & 0.12 & 2 \\
\hline 07 & L 1642 & 0.48 & 0.30 & 0.23 & 0.14 & 0.10 & - & 0.11 & \\
\hline 08 & L 1642 & 0.48 & 0.32 & 0.21 & 0.14 & 0.12 & - & 0.11 & \\
\hline 09 & L 1642 & 0.50 & 0.32 & 0.24 & 0.16 & 0.11 & - & 0.10 & \\
\hline 10 & L 1642 & 0.50 & 0.34 & 0.23 & 0.14 & 0.11 & - & 0.12 & 2 \\
\hline 11 & L 1642 & 0.52 & 0.34 & 0.24 & 0.15 & 0.10 & - & 0.15 & \\
\hline
\end{tabular}

1. Standard La Silla extinction. $\quad$ 2. Average extinction for 1988-12-4/7/8/9/11

b) $B V R I$ observations

\begin{tabular}{|r|l|rrrr|l|}
\hline \multicolumn{1}{|c|}{ Date } & Targets & \multicolumn{4}{|c|}{ Extinction coefficents } & Remarks \\
& observed & $V$ & $B-V$ & $V-R$ & $V-I$ & \\
\hline 1978-02-05 & A1060 & 0.13 & 0.11 & 0.06 & 0.10 & 1 \\
06 & A496, A1060 & 0.13 & 0.11 & 0.06 & 0.10 & \\
07 & A496, A1060, A1146 & 0.13 & 0.13 & 0.05 & 0.08 & \\
08 & A496, A1146, A1060 & 0.13 & 0.11 & 0.06 & 0.10 & 1 \\
\hline
\end{tabular}

1. Extinction adopted from 1978-02-06

c) uvgr observations

\begin{tabular}{|c|c|c|c|c|c|c|}
\hline \multirow[t]{2}{*}{ Date } & \multirow{2}{*}{$\begin{array}{l}\text { Targets } \\
\text { observed }\end{array}$} & \multicolumn{4}{|c|}{ Extinction coefficents } & \multirow[t]{2}{*}{ Remarks } \\
\hline & & $u$ & $v$ & $g$ & $r$ & \\
\hline 1980-05-10 & A1146 & 0.50 & 0.30 & 0.13 & - & 1 \\
\hline 11 & A1146 & 0.50 & 0.30 & 0.13 & - & 1 \\
\hline $1982-02-24$ & A1146 & 0.55 & 0.34 & 0.17 & 0.11 & 2 \\
\hline 25 & $\mathrm{~A} 754, \mathrm{~A} 1146$ & 0.55 & 0.34 & 0.17 & 0.11 & 2 \\
\hline 26 & $\mathrm{~A} 754, \mathrm{~A} 1146$ & 0.55 & 0.34 & 0.17 & 0.11 & 2 \\
\hline 27 & $\mathrm{~A} 754, \mathrm{~A} 1146$ & 0.55 & 0.34 & 0.17 & 0.11 & 2 \\
\hline 28 & A1146 & 0.55 & 0.34 & 0.17 & 0.11 & 2 \\
\hline 03-01 & A1146 & 0.55 & 0.34 & 0.17 & 0.11 & \\
\hline $08-15$ & CL004.8-3450 & 0.58 & 0.29 & 0.17 & 0.07 & \\
\hline 16 & CL004.8-3450 & 0.61 & 0.29 & 0.17 & 0.08 & \\
\hline $1983-01-13$ & A1146 & 0.43 & 0.24 & 0.13 & 0.09 & 3 \\
\hline 14 & A754 & 0.43 & 0.24 & 0.13 & 0.09 & 3 \\
\hline 15 & $\mathrm{~A} 754, \mathrm{~A} 1146$ & 0.43 & 0.24 & 0.13 & 0.09 & 3 \\
\hline 16 & $\mathrm{~A} 754, \mathrm{~A} 1146$ & 0.43 & 0.24 & 0.13 & 0.09 & 3 \\
\hline 17 & $\mathrm{~A} 754, \mathrm{~A} 1146$ & 0.43 & 0.24 & 0.13 & 0.09 & 3 \\
\hline
\end{tabular}

1. Average extinction for 1980-05-10/11. 2. Extinction adopted from 1982-03-01

3. Average extinction for $1983-01-13$ to 17 
given in Table $2 \mathrm{~b}$. The $B$ and $V$ magnitudes and colours are in the Johnson (1955) system and the $V-R$ and $V-I$ colours in the Arizona-Tonanzintla system (Iriarte et al. 1965). The following type of colour equations (with the values of constants for Feb. 5th inserted) were used for the colour transformations between the extraterrestrial instrumental magnitudes, $b, v, r, i$, and the standard $U, B, V, R, I$ magnitudes:

$$
\begin{aligned}
B-V & =((b-v)+0.671) / 0.947 \\
V & =v+4.306 \\
V-R & =((v-r)-0.490) / 1.387 \\
V-I & =((v-i)+0.910) / 1.070
\end{aligned}
$$

These equations represent a simplified form of the system used for stellar photometry. Colour-terms depending on stellar spectral characteristics (such as the $U-B$ excess) were not included since the night sky spectrum is very different from typical stellar spectra.

The sky brightness values are expressed in units of 10th magnitude stars per square degree, $S_{10}$. The transformation into the frequently-used units of mag/ $\square$ " is given by $I\left(\mathrm{mag} / \square^{\prime \prime}\right)=-2.5 \lg \mathrm{I}\left(\mathrm{S}_{10}\right)+27.78$.

\section{3. uvgr data}

The sky brightness measurements in the uvgr intermediate-band photometric system of Thuan \& Gunn (1976) were carried out in 16 nights during four observing sessions: May 10-11 1980, February 24 - March 1 1982, August 15-16 1982, and January 13-18 1983. The aim of the observations was to measure the diffuse intergalactic light in clusters of galaxies (see Schnur 1980). Simultaneous measurements were carried out at the ESO 1-m and 50-cm telescopes. The standard ESO filters for the uvgr system (Nos. 232-235 for the 1-m and 236-239 for the 50-cm telescope) and RCA 31034A photomultipliers were used. The diaphragm size at the 1-m telescope was 86.55 arcsec, the integration time per filter was $10 \mathrm{~s}$, and the minimum count rates were $\sim 2000$ counts/s. A "beam efficiency" of 0.91 was used, i.e. the effective aperture area was the same as for the intermediate band observations.

The sky brightness measurements were calibrated and atmospheric extinction coefficients (see Table 2c) were determined by means of standard star observations from the list of Thuan \& Gunn (1976). Our photometric system was found to reproduce the Thuan and Gunn standard system with sufficient accuracy; thus, no colour transformations were introduced. The sky brightness values are expressed, as for $B V R I$, in $S_{10}$ units.

The zero points for the magnitudes and colour indices in the uvgr-system are defined wrt the primary standard $\mathrm{BD}+17^{\circ} 4708$, an sdF6 star. In order to facilitate a comparison with sky brightness data in the $U B V R I$-system we present the uvgr data also in a modified uvgr system in which the zero points are defined wrt A0V spectral type. Using the average values for six A0V stars as observed by Kent (1985) we find that an A0V star with $U=B=V=R=0$ has the following uvgr magnitudes: $u=-0.24, v=-0.54, g=-0.19, r=0.32$.

Thus, in order to transform the sky brightnesses into an "A0V-based uvgr-system" we have to multiply the uvgr intensities (given in $S_{10}$ ) by the factors:

$0.802(u), 0.608(v), 0.839(g)$, and $1.343(r)$.

To facilitate a comparison with our intermediate band photometry we have calibrated the uvgr measurements in units of $10^{-9} \mathrm{ergs} \mathrm{cm}^{-2} \mathrm{~s}^{-1}$ sterad $^{-1} \AA^{-1}$. This was done by using the spectrophotometry of Oke and Gunn (1983) for the uvgr primary standard $\mathrm{BD}+17^{\circ} 4708$ along with the uvgr filter band pass curves as given by Thuan \& Gunn (1976). Thus we found the following factors by which the uvgr intensities given in $S_{10}$ are to be multiplied to convert them into units of $10^{-9} \mathrm{ergs} \mathrm{cm}^{-2} \mathrm{~s}^{-1}$ sterad $^{-1}$ $\AA^{-1}: 1.08(u), 1.39(v), 1.41(g)$, and $1.00(r)$.

\section{Results}

\subsection{Sky brightness values: Intermediate band data}

The individual intermediate-band sky brightness values for each night are presented in Fig. 3a. The data were taken only during moonless hours (except at the end of the nights 1987-12-15 and 1988-12-02) and mostly with no twilight contamination. In a few cases we have included some measurements during the evening twilight hours: 1980-12-07/09, 1988-12-08/09/10. In these cases one can see an upturn of sky brightness, especially at the shortest wavelengths (see Fig. 3a).

We present in Table 3 and in Fig. 5 the nightly averages of the sky brightnesses. A representative value for each observing night was determined by averaging the values between 15 and 17 hours LST in the year 1979 (target: L134) and between 3 and 6 hours LST for the other years (target: L1642). For the night 1988-12-06 we use for the averaging only the last hour of the night (during which L1642 was observed). The whole night of 1988-12-05 was spent at a different position (S245) (See Fig. 3).

\subsection{Sky brightness values: BVRI data}

The individual $B V R I$ sky brightness values along with the colour indices $B-V, V-R$, and $R-I$ are presented in in Fig. 3b. The solid and open symbols in this figure refer to ON- and OFF- positions, respectively, in the observed clusters of galaxies. The nightly averages of sky brightness are given in Table 5 both in units of $S_{10}$ and mag/ $\square^{\prime \prime}$.

\subsection{Sky brightness values: uvgr data}

The individual sky brightness measurements in the uvgr system are shown in Fig. 3c for the three nights Feb. 24, 26, and 28 1982. The colour indices $u-v, v-g$, and $g-$ $r$ are shown as well. The nightly averages are given in 
Table 3. Average sky brightnesses as observed for individual nights in the intermediate bandpasses

\begin{tabular}{|r|rcccccc|}
\hline & $I(u)$ & $I(3840)$ & $\begin{array}{c}I(4160) \\
{\left[10^{-9}\right.}\end{array} \mathrm{erg} \mathrm{s}^{-1}$ & $\begin{array}{c}I(b) \\
\mathrm{cm}^{-2}\end{array}$ & $\begin{array}{c}I(\mathrm{H} \beta) \\
\left.\mathrm{str}^{-1} \AA^{-1}\right]\end{array}$ & $I(\mathrm{OIII})$ & $I(y)$ \\
\hline $1979-06-22$ & - & 246 & 247 & 293 & - & - & 445 \\
23 & - & 248 & 252 & 303 & - & - & 456 \\
24 & - & 248 & 253 & 307 & - & - & 518 \\
$1980-12-06$ & 553 & 353 & 381 & 376 & - & - & 816 \\
07 & 402 & 260 & 308 & 334 & - & - & 585 \\
08 & 469 & 299 & 347 & 386 & - & - & 773 \\
09 & 348 & 252 & 285 & 299 & - & - & 517 \\
10 & 378 & 285 & 316 & 331 & - & - & 616 \\
$1987-12-15$ & 184 & 160 & 194 & 207 & - & - & 279 \\
16 & 163 & 149 & 189 & 203 & - & - & 243 \\
17 & 203 & 178 & 211 & 214 & - & - & 313 \\
18 & 227 & 190 & 224 & 224 & - & - & 327 \\
19 & 182 & 151 & 191 & 208 & - & - & 260 \\
20 & 210 & 180 & 214 & 222 & - & - & 320 \\
$1988-12-02$ & 279 & 210 & 250 & 253 & 261 & 244 & 419 \\
03 & 399 & 292 & 306 & 291 & 292 & 237 & 648 \\
04 & 325 & 226 & 259 & 268 & 312 & 289 & 522 \\
05 & 454 & 336 & 377 & 359 & 477 & 357 & 690 \\
06 & 348 & 269 & 299 & 284 & 301 & - & 573 \\
07 & 287 & 218 & 262 & 268 & 278 & - & 451 \\
08 & 263 & 200 & 244 & 253 & 255 & - & 415 \\
09 & 274 & 202 & 251 & 269 & 284 & - & 449 \\
10 & 228 & 186 & 233 & 257 & 267 & - & 374 \\
11 & 257 & 200 & 257 & 285 & 304 & - & 452 \\
\hline & & & & & & &
\end{tabular}

Table 4. Synthetic broad band sky brightnesses for $U B V$ as derived from the intermediate band data of Table 3

\begin{tabular}{|r|rrr|rcc|}
\hline & $I_{U}$ & $I_{B}$ & $I_{V}$ & $I_{U}$ & $I_{B}$ & $I_{V}$ \\
& & {$\left[S_{10}\right]$} & & \multicolumn{3}{|c|}{$\left[\mathrm{mag} /{ }^{\prime \prime}\right]$} \\
\hline $1979-06-22$ & - & 128 & 354 & - & 22.51 & 21.41 \\
23 & - & 131 & 363 & - & 22.48 & 21.38 \\
24 & - & 133 & 409 & - & 22.47 & 21.25 \\
$1980-12-06$ & 351 & 184 & 634 & 21.41 & 22.11 & 20.77 \\
07 & 257 & 152 & 461 & 21.75 & 22.32 & 21.12 \\
08 & 298 & 175 & 604 & 21.59 & 22.17 & 20.83 \\
09 & 229 & 139 & 407 & 21.88 & 22.42 & 21.25 \\
10 & 252 & 155 & 483 & 21.78 & 22.30 & 21.07 \\
$1987-12-15$ & 128 & 93 & 224 & 22.51 & 22.85 & 21.90 \\
16 & 116 & 90 & 197 & 22.62 & 22.89 & 22.04 \\
17 & 142 & 100 & 250 & 22.40 & 22.78 & 21.78 \\
18 & 156 & 105 & 261 & 22.29 & 22.72 & 21.74 \\
19 & 125 & 92 & 210 & 22.53 & 22.87 & 21.97 \\
20 & 146 & 102 & 256 & 22.37 & 22.75 & 21.76 \\
03 & 186 & 119 & 331 & 22.10 & 22.59 & 21.48 \\
04 & 263 & 146 & 503 & 21.73 & 22.36 & 21.03 \\
05 & 212 & 126 & 408 & 21.96 & 22.53 & 21.25 \\
06 & 2301 & 177 & 540 & 21.58 & 22.16 & 20.95 \\
07 & 192 & 125 & 447 & 21.86 & 22.40 & 21.15 \\
08 & 176 & 117 & 328 & 22.07 & 22.53 & 21.40 \\
09 & 182 & 122 & 355 & 22.13 & 22.56 & 21.40 \\
10 & 156 & 114 & 299 & 22.29 & 22.64 & 21.59 \\
11 & 173 & 126 & 358 & 22.18 & 22.52 & 21.39 \\
\hline & & & & & &
\end{tabular}


Table 5. Average sky brightnesses for individual nights as measured in the $B V R I$ system

\begin{tabular}{|c|c|c|c|c|c|c|c|c|}
\hline & $I_{B}$ & & $I_{R}$ & $I_{I}$ & \multicolumn{4}{|c|}{$\begin{array}{c}I_{V} \quad I_{R} \\
{\left[\mathrm{mag} / \square^{\prime \prime}\right]}\end{array}$} \\
\hline 1978-02-05 & 109 & 284 & 630 & 1944 & 22.69 & 21.64 & 20.78 & 19.56 \\
\hline 06 & 98 & 267 & 625 & 2098 & 22.80 & 21.71 & 20.79 & 19.48 \\
\hline 07 & 95 & 234 & 574 & 2111 & 22.84 & 21.86 & 20.88 & 19.47 \\
\hline 08 & 88 & 250 & 642 & 2201 & 22.92 & 21.79 & 20.76 & 19.42 \\
\hline
\end{tabular}

Table 6. Average sky brightnesses for individual nights as measured in the uvgr system. The primed values in the right-hand part of the table are sky brightnesses converted to a system where the zero points are wrt spectral type A0V

\begin{tabular}{|r|cccc|cccr|}
\hline & $I_{u}$ & $I_{v}$ & $I_{g}$ & $I_{r}$ & $I_{u}^{\prime}$ & $I_{v}^{\prime}$ & $I_{g}^{\prime}$ & $I_{r}^{\prime}$ \\
& \multicolumn{3}{|c}{$\left[S_{10}\right]$} & & \multicolumn{4}{c|}{$\left[\mathrm{mag} / \square^{\prime \prime}\right]$} \\
$1980-05-10$ & 243 & 175 & 225 & - & 22.06 & 22.71 & 22.09 & - \\
11 & 276 & 190 & 222 & - & 21.92 & 22.62 & 22.10 & - \\
$1982-02-24$ & 276 & 183 & 214 & 464 & 21.92 & 22.66 & 22.14 & 20.79 \\
25 & 344 & 222 & 236 & 536 & 21.68 & 22.45 & 22.04 & 20.64 \\
26 & 448 & 265 & 271 & 546 & 21.39 & 22.26 & 21.89 & 20.62 \\
27 & 343 & 213 & 248 & 570 & 21.68 & 22.50 & 21.98 & 20.57 \\
28 & 238 & 175 & 200 & 534 & 22.08 & 22.71 & 22.22 & 20.64 \\
$03-01$ & 330 & 227 & 242 & 487 & 21.72 & 22.43 & 22.01 & 20.74 \\
$08-15$ & 206 & 181 & 197 & 550 & 22.23 & 22.68 & 22.23 & 20.61 \\
16 & 238 & 181 & 197 & 528 & 22.08 & 22.68 & 22.23 & 20.65 \\
$1983-01-13$ & 195 & 149 & 179 & 327 & 22.29 & 22.89 & 22.34 & 21.17 \\
14 & 276 & 198 & 213 & 406 & 21.92 & 22.58 & 22.15 & 20.94 \\
15 & 193 & 148 & 169 & 379 & 22.31 & 22.89 & 22.40 & 21.01 \\
16 & 285 & 189 & 202 & 383 & 21.88 & 22.63 & 22.21 & 21.00 \\
17 & 218 & 160 & 169 & 390 & 22.17 & 22.81 & 22.40 & 20.98 \\
\hline
\end{tabular}

Table 6 for all 15 uvgr observing nights. The left-hand part of the table gives the data in $S_{10}$ units in the proper uvgr system, whereas the right-hand part contains the sky brightnesses in a modified system where - in order to facilitate comparison with $U B V R I$ data - the zero points are wrt A0V spectral type (see Sect. 2.3).

\subsection{Sky brightness variations}

As the zenith distance of a measuring position changes during the night one would expect that its brightness varies, because of the varying path length through the airglow layer, in accordance with the van Rhijn (1921) formula, i.e. very roughly speaking proportionally to the airmass. This would mean that for our targets a minimum sky brightness should be observed at LST $=16 \mathrm{~h}$ for L134 and at LST $=4.5 \mathrm{~h}$ for L1642. Such a systematic behaviour is, however, generally not observed. The sky brightness variations are dominated by the overall variability of the airglow and possibly also by its patchy structure.

The sky brightness variations in individual nights display a multitude of different patterns, with both increasing, decreasing and almost constant time behaviour (see
Fig. 3). We do not see any indications for the systematic every-night behavior of a decreasing sky brightness after the end of twilight as reported by Walker (1988) for the San Benito Mountain during the period 1976-87. For Kitt Peak, Pilachowski et al. (1989) also found greatly varying brightness vs. time behaviour patterns.

It can be seen from Fig. 3a that in most cases the time variations in different intermediate band filters seem to be correlated. In order to study the correlations in more detail we have calculated the correlation coefficients between the different colours for each night. The median values of these correlation coefficients are shown in Table 7. It can be seen that large correlation coefficients $(\rho \sim 0.9)$ are found between all band passes. We present in Fig. 4 examples of the correlations between the different filters with the $y$-filter for the years 1979, 1980 and 1987. We see that in the 1980 and 1987 observing periods the correlations were tight, both during each individual night and from night to night, whereas in 1979 the scatter was much larger. For our broad band (BVRI) measurements in 1978 February the correlations between the different filters are less pronounced. This is especially true for the $R$ and $I$ bands and for the nights1978-02-7/8 (see Fig. 3b). 
Barbier (1956) investigated extensively the correlations between the different airglow emission lines and "pseudocontinuum" bands using eight intermediate bands centered at $3670 \AA(\Delta \lambda=350 \AA), 4400 \AA(570 \AA)$, $5260 \AA(120 \AA), 5580 \AA(115 \AA), 5900 \AA(140 \AA), 6340 \AA$ $(460 \AA), 6300 \AA(130 \AA)$ and $6700 \AA$ (broad band). Three filters of his set correspond closely to our filters $u(3500 \AA)$, $b(4670 \AA)$, and $y(5470 \AA)$. Barbier found good correlations between several of his eight band passes both within one night and from night to night. He established three "covariance groups" of emissions. Barbier's first group is of special interest for us. It includes the four bandpasses 3670 $\AA, 4400 \AA, 5260 \AA$ and $5580 \AA$. We do not have a filter corresponding to $5260 \AA$, but we find that the other three band passes corresponding to Barbier's group I also show good correlation in our case.

Dumont (1965) has utilized extensively the correlation of the $5577 \AA$ line with the "pseudocontinuum" at $4600 \AA(\Delta \lambda=200 \AA), 5020 \AA(240 \AA)$, and $5700 \AA$ $(140 \AA)$ for the purpose of separating the airglow from the ZL and other extraterrestial night sky components.

Table 7. Median correlation factors of intensities over all observed nights

\begin{tabular}{|c|rrrrr|}
\hline$\rho_{i j}$ & $u$ & 3840 & 4160 & $b$ & $y$ \\
\hline$u$ & & 0.84 & 0.94 & 0.92 & 0.93 \\
385 & 0.84 & & 0.94 & 0.87 & 0.91 \\
415 & 0.94 & 0.94 & & 0.94 & 0.92 \\
$b$ & 0.92 & 0.87 & 0.94 & & 0.95 \\
$y$ & 0.93 & 0.91 & 0.92 & 0.95 & \\
\hline
\end{tabular}

\section{Correlation of the night sky brightness with solar activity}

Rayleigh (1928) and Rayleigh \& Jones (1935) reported a correlation between the intensity of the $5577 \AA[\mathrm{OI}]$ airglow line with sunspot numbers. This correlation was questioned later (e.g. Roach 1955, for a review see Chamberlain 1961) but is now well established both for the $5577 \AA$ as well as other emission lines of [OII], Na D and $\mathrm{OH}$ (see e.g. Rosenberg \& Zimmerman 1967, and Walker 1988 for a review). Walker (1988) found that during the period 1976-87 the broad band night-sky brightnesses in the $V$ and $B$ photometric bands were well-correlated with the solar $10.7 \mathrm{~cm}$ radio flux, used as an indicator of the solar activity. These results demonstrated that the correlation between sky brightness and solar activity exists, not only for the strong lines but also for the airglow (pseudo)continuum emission which dominates at least in the $B$ band.

We present in Fig. 5 the nightly averages as a function of date for our intermediate-band and uvgr observations. In the figure are also plotted the monthly mean values of the $10.7 \mathrm{~cm}$ solar flux (in $10^{4} \mathrm{Jy}$ ) and the sunspot numbers. The $u$-bands of both these photometric systems are very similar (see Fig. 2); therefore, these data have been combined into the same figure. The Thuan \& Gunn $v$-band is well covered by the combination of our 3840 and $4160 \AA$ filters; therefore, we have combined the $v$-band data with average values of the 3840 and $4160 \AA$ intensities. The Strömgren $b$ band covers the short-wavelength half of the Thuan \& Gunn $g$-band; because the $f_{\lambda}$ - spectrum of the night sky is almost flat over this wavelength domain (see Fig. 7 of this paper and Fig. 7 of Leinert et al. 1995), we have combined the $b$ and $g$ band data into the same figure. We note also that our targets for the intermediate band observations (L134 and L1642) and the uvgr-observations (clusters of galaxies) were at almost the same ecliptic latitude and had nearly the same $I_{\mathrm{ZL}}$ (see Table 1 ) and the same airmass of $\sim 1-1.4$ (see Fig. 1).

It can be seen from Fig. 5 that the sky brightness shows a slight rise from 1979 to 1980 and then a clear tendency of decreasing intensity to 1987, and finally an increase to the year 1988. This dependence has a clear similarity with the solar activity: solar activity had its maximum in December 1979 and a minimum in September 1986; the next maximum was in July 1989. However, we see also that the scatter between the individual nights within a given observing period, particularly at the times of maximum, is roughly half of the long term (year-to-year) changes.

In order to study in more detail the correlation with solar activity we plot, in Fig. 6, the nightly averages of sky brightness against the solar $10.7 \mathrm{~cm}$ flux. The error bars indicate the standard deviations of individual measured values (due to nightly airglow variations) included in the averages. The solar $10.7-\mathrm{cm}$ flux values, as measured at 17.00 hours UT every day at the Ottawa River Solar Observatory, were obtained from National Geophysical Data Center, Boulder, Co. They are the "observed values" (i.e. not corrected to a $1 \mathrm{AU}$ solar distance). For each night the flux value from the day before the night was adopted. It has been found previously that the $10.7 \mathrm{~cm}$ flux is a good indicator of the solar activity and has a somewhat better correlation with the $5577 \AA$ airglow line than the Zürich sunspot numbers (e.g. Rosenberg \& Zimmerman 1967).

Correlation coefficients for the individual scatter diagrams are presented in Table 8 . It can be seen that the two quantites are clearly correlated in all filters.

The least squares fits are also given in Table 8. The slopes of these relationships are such that, for our observed range of solar flux values from 90 to $19010^{4} \mathrm{Jy}$, the sky brightness changes by a factor of $1.69,1.50,1.49$, $1.46,1.45,2.06$, and 1.55 in the colours $u, 3840,4160$, $v /(3840+4160), b / g, y$, and $r$, respectively. A comparison with the results of Leinert et al. (1995) shows that the slopes are, in the present case, somewhat larger in the $u, 3840 \AA$ and $4160 \AA$ bands, and somewhat smaller in the $b / g$ band than the coresponding values for Calar 

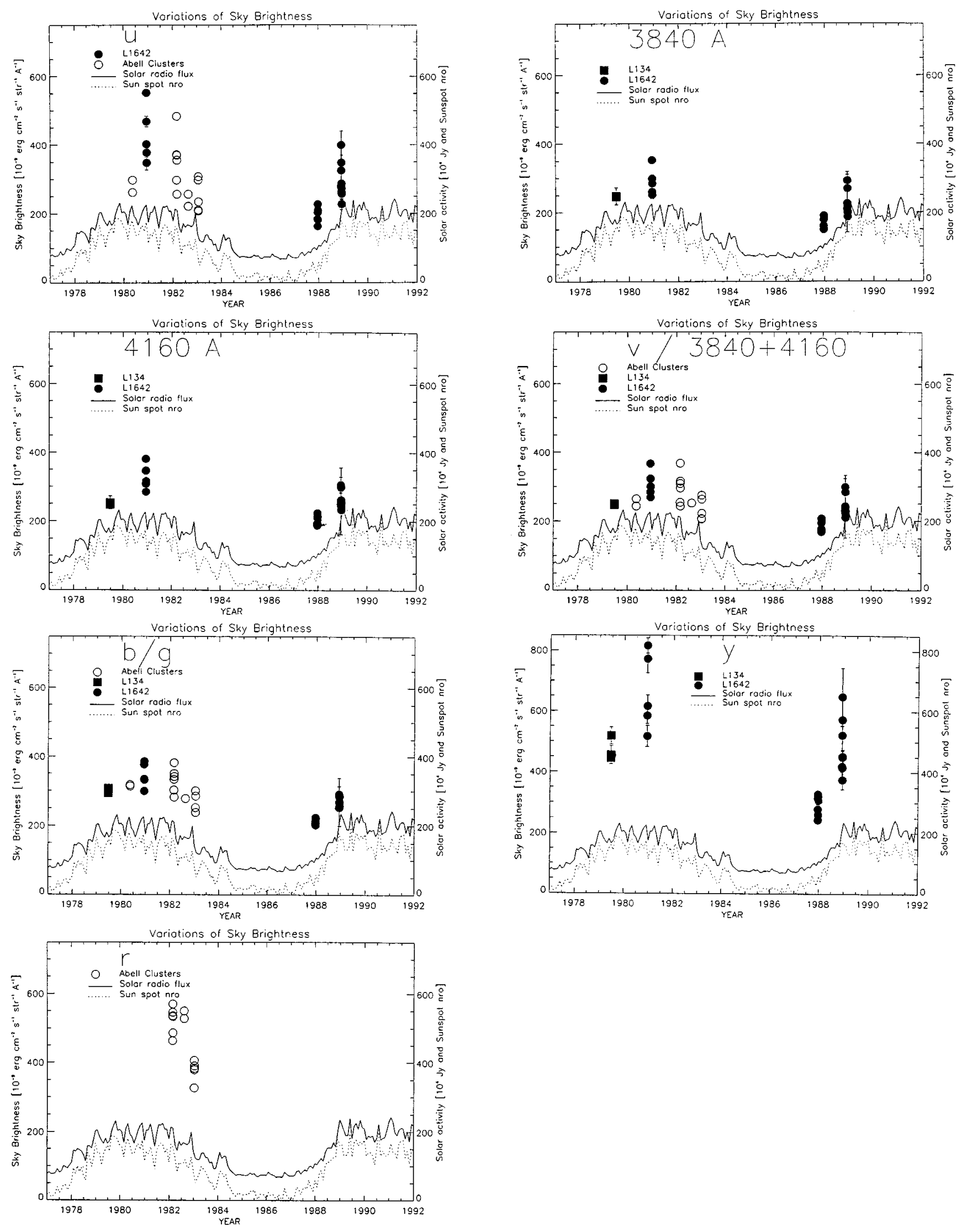

Fig. 5. Nightly averaged sky brightnesses (unit: $10^{-9} \mathrm{erg} \mathrm{cm}^{-2} \mathrm{~s}^{-1}$ sterad $^{-1} \AA^{-1}$ ) vs. date for the intermediate- (filled circles) and uvgr-band (open circles) data The numbers on the time axis denote the beginning of the year. The solar cycle, in terms of sunspot numbers (dashed line) and solar 10.7-cm flux (solid line, unit: $10^{4} \mathrm{Jy}$ ), is also shown. The values are monthly mean values 

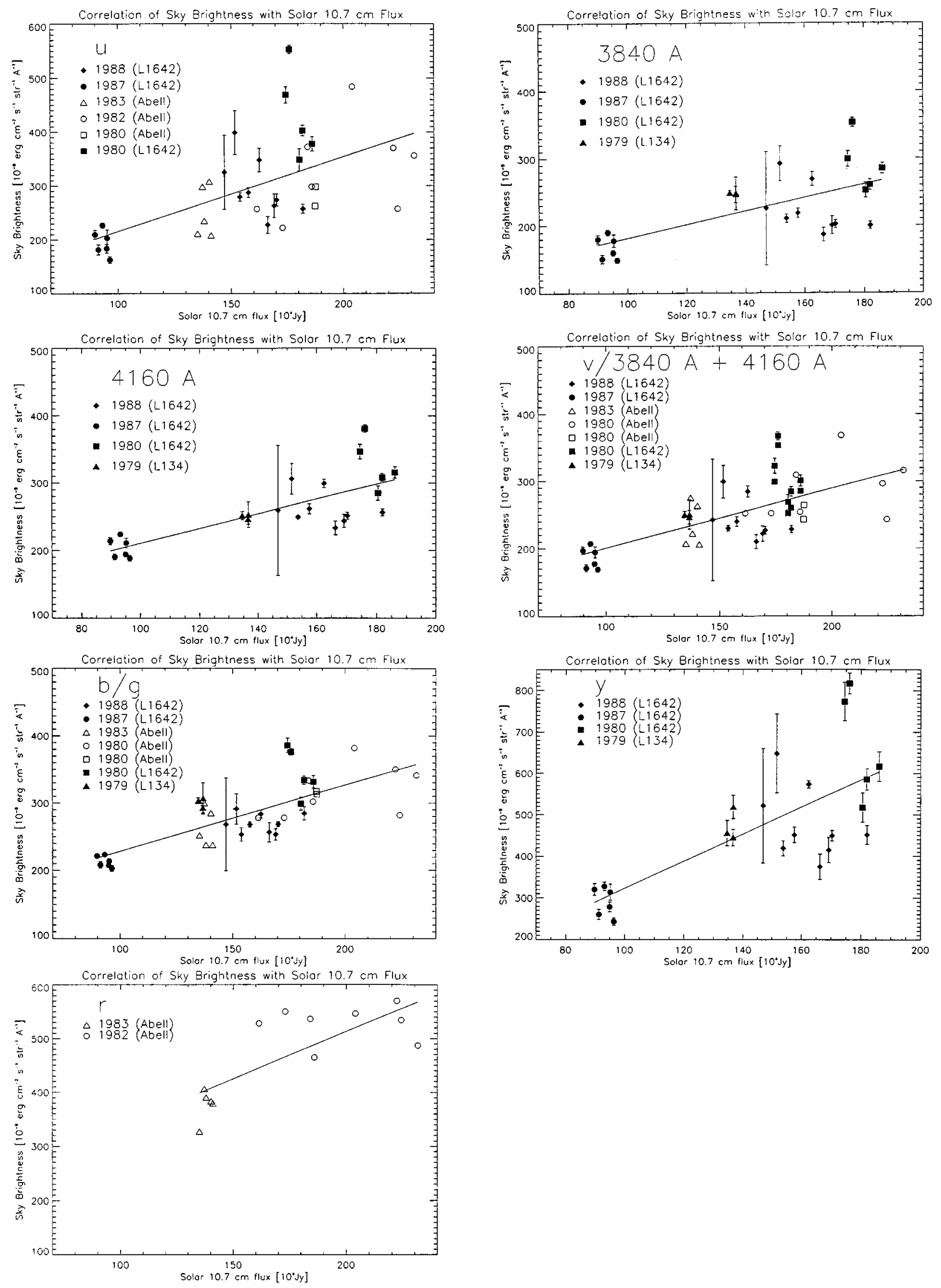

Fig. 6. Correlation of the night-sky brightness (unit: $10^{-9} \mathrm{erg} \mathrm{cm}^{-2} \mathrm{~s}^{-1} \operatorname{sterad}^{-1} \AA^{-1}$ ) with the solar $10.7-\mathrm{cm}$ flux density (unit: $10^{4} \mathrm{Jy}$ ) for the intermediate- (filled symbols) and uvgr-band (open symbols) data 
Table 8. Correlation of the night sky brightness with the solar $10.7 \mathrm{~cm}$ flux. The correlation coefficients $\rho$ and the least squares fit parameters $\left(I_{\mathrm{sky}}=a \cdot f_{\mathrm{sol}}+b\right)$ are given for each colour. $I_{\text {sky }}$ is in $10^{-9} \mathrm{ergs} \mathrm{cm}^{-2} \mathrm{~s}^{-1} \mathrm{sterad}^{-1} \AA^{-1}$ and $f_{\text {sol }}$ is in $10^{4} \mathrm{Jy}$

\begin{tabular}{|l|rrrrrrr|}
\hline \multicolumn{2}{|c}{$u$} & 3840 & 4160 & $v / 3840 / 4160$ & $b / g$ & $y$ & $r$ \\
\hline$\rho$ & 0.58 & 0.66 & 0.76 & 0.69 & 0.76 & 0.73 & 0.78 \\
$a$ & 1.39 & 0.99 & 1.10 & 0.89 & 0.98 & 3.26 & 1.76 \\
$b$ & 77 & 82 & 101 & 112 & 130 & -3 & 160 \\
\hline
\end{tabular}

Alto. The mean value of the slopes for $3840,4160, v$ and $b$ corresponds to $0.41 \mathrm{mag}$., in good agreement with the difference of $0.35-0.40 \mathrm{mag}$ which is obtained from Walker's (1988) $B$ band data (his Fig. 4). Our corresponding number for the $y$ band data is 0.79 magnitudes which is larger than Walker's $V$ band value of $\sim 0.45 \mathrm{mag}$; this is understandable since the $y$ band sky brightness is strongly influenced by the $5577 \AA$ line. Our number for the Thuan \& Gunn $r$ band is $0.48 \mathrm{mag}$, which is in good agreement with Walker's $V$ value. Our observations thus confirm both qualitatively and quantitatively Walker's (1988) results on the correlation of the airglow "pseudocontinuum" intensity with the solar activity. A corollary of this correlation is that, for the purpose of comparing sky brightnesses obtained at different dates, one has to take the level of solar activity into account.

We have also checked whether the correlation found for the year-to year variations pertains also to the short-term (night-to-night) variations of the sky brightness. For this purpose the data points for different years are indicated with different symbols in Fig. 6. The data points for each year were obtained during an observation period of about one week. As can be seen from Fig. 6, the daily correlations are much weaker than the overall correlation. In the years 1979 and 1987 (nearest to the solar activity minima) both the sky brightness and solar flux remained comparatively stable. In the years 1980 and 1988 there is more scatter in the solar flux values but if there is any trend in the sky brightness values, it is in the opposite direction to the expected one. We conclude that our data do not indicate any conclusive short-term correlation between the nightsky continuum brightness and the solar $10.7 \mathrm{~cm}$ flux as measured during the preceeding day. Walker's (1988) suggestion that the night sky brightness could be predicted by using the solar radio flux measurement of the previous day appears too optimistic on the basis of our data. Our results are in qualitative agreement with Rosenberg \& Zimmerman (1967) who found that the correlation between the $5577 \AA$ line and the $10.7 \mathrm{~cm}$ flux was much weaker for the nightly averages than for the monthly averages.

Besides the solar activity also the lunar and solar tides have been found to trigger variations in the airglow emission line intesities (cf. Chamberlain 1961, p. 548; Christophe-Glaume 1965; Hecht et al. 1995). Inspection of our data for such effects did not reveal any clear indications for a tidal modulation.

\section{Comparison with other observing sites}

\subsection{Level of sky brightness: intermediate band data}

The results of the previous section demonstrate that it is important to take the level of solar activity into account before trying to compare sky brightness measurements made at different sites at different times. Also, even at the same level of solar activity (10.7 cm flux) large nightto-night variations occur in the sky brightness (especially close to solar maxima) making it necessary to collect data during several nights before any conclusions can be drawn.

We can compare our measured intermediate band sky brightnesses directly with Barbier's $(1956,1961)$ results from Haute Provence in the years 1953-59, with Dachs's(1970) results from the "Jonathan Zenneck" research station at Tsumeb (Namibia) in the year July 1965 to June 1966, and the sky brightness measurements from Calar Alto in the years 1989-1993 (Leinert et al. 1995). We compare in Table 9 our data with Barbier, Dachs and Leinert et al. in four wavelength domains which were close to our's. For each data set (exept Tsumeb) we give the minimum and maximum value determined as the average of the three smallest (largest) values in the data set. For Tsumeb the values for individual nights were not available but only the mean values (for the whole data set) and their standard errors; they are given in Table 9. It can be seen that (1) the range of the ultraviolet values $(3670 / 3500$ $\AA$ ) is similar in Haute Provence and Calar Alto, but the ESO minimum values are lower by $\sim 20$ per cent; (2) at $3920 \AA$ the Tsumeb value is $\sim 20 \%$ lower than the ESO minimum value at $3840 \AA$, but it should be noted that for Tsumeb the contribution of the airglow line at $3914 \AA$ has been subtracted and that the whole Tsumeb data set refers to the time of solar minimum; the Calar Alto minimum value is $\sim 20 \%$ higher than the ESO value whereas the maximum values for these two sites are almost the same; (3) for the blue filters $(4400 / 4670 / 4900 \AA)$ the ESO values agree very well with Haute Provence and Tsumeb, while the minimum values of Calar Alto are $\sim 30 \%$ higher; (4) for the green/yellow filters it is more difficult to compare the ESO results with the three other data sets because the Haute Provence and Calar Alto filters avoid the 
$5577 \AA$ line and in the case of Tsumeb its contribution has been subtracted; nevertheless, the minimum ESO values are close to the minimum values for Calar Alto and Tsumeb and no more than $35 \%$ higher than the values for Haute Provence which can be accounted for by the $5577 \AA$ line; (5) in summary, although the data sets are not fully comparable - e.g. reduction to zenith would reduce the ESO and Calar Alto values by $10-15 \%$ - the fact remains that the ESO ultraviolet sky is darker by perhaps $20-30 \%$ and and the blue/green sky about equal to Haute Provence as in 1953-59. The sky is darker at La Silla than in Calar Alto probably because of (modest) light pollution at Calar Alto emanating from Almeria.

\subsection{Level of sky brightness: $B$ and $V$}

Most sky brightness measurements available in the literature have been carried out in the broad band filters $B$ and $V$.

We can directly compare our data with them only for the four nights 1978-02-05 to -08. In order to compare our intermediate-band data with the $B$ and $V$ data we have estimated the conversion factors using the $U X, B$, and $V$ bandpass curves of Bessel (1990). We find the following transformation formulae:

$$
\begin{aligned}
I_{U}\left[S_{10}\right] & =0.471 \cdot I_{u}+0.231 \cdot I_{3840}+0.026 \cdot I_{4160} \\
I_{B}\left[S_{10}\right] & =0.059 \cdot I_{3840}+0.215 \cdot I_{4160}+0.185 \cdot I_{b} \\
& +0.015 \cdot I_{y} \\
I_{V}\left[S_{10}\right] & =0.097 \cdot I_{b}+0.733 \cdot I_{y},
\end{aligned}
$$

where the intermediate band data are, as throughout this paper, in units of $10^{-9}$ ergs $\mathrm{cm}^{-2} \mathrm{~s}^{-1}$ sterad $^{-1} \AA^{-1}$.

We should note that although our intermediate band filters cover only about half of the total wavelength range of the $B$ filter they still provide a reasonably good coverage of this band pass. The $y$ filter is centered at almost the same wavelength as the $V$ filter but is narrower by a factor of $\sim 5$ and is, therefore, much more strongly affected by the bright airglow line at $5577 \AA$. Therefore, our estimated values for the $V$ band, which are based almost solely on the $y$ observations, represent only an upper limit to the sky brightness in $V$.

We present in Table 4 our synthetic $U B V$ sky brightness values and in Table 5 the observed ones. A direct comparison of the synthetic values with the measured ones is not possible since we did not have measurements in both photometric systems during the same night or even within the same observing period. We can do some comparisons, however, because the solar activity level was, during our $B V R I$ observations in 1979 February, similar as the average value for our 1987 and 1988 observing periods. The average sky brightnesses in these two periods were:

1978 Feb.: $I_{B}=22.81, I_{V}=21.75 \mathrm{mag} / \square^{\prime \prime}$

1987 Dec. $/ 1988$ Dec.: $I_{B}=22.65, I_{V}=21.59 \mathrm{mag} / \square^{\prime \prime}$

where the latter values are synthetic ones. We have estimated the contribution of the $5577 \AA$ line to the signal in the $y$ filter by assuming for its intensity a range from 40 to 400 rayleigh (see e.g. Dumont 1965; Dachs 1968) and for the continuum an intensity range as observed in this study. The result is 0.16 to $0.76 \mathrm{mag} / \square^{\prime \prime}$. Similarly, the contribution to the $V$ band is estimated to be 0.05 to 0.25 $\mathrm{mag} / \square^{\prime \prime}$. Massey et al. (1990), using the measured spectral energy distribution of the night sky at Kitt Peak during the nights 1988-02-18 and 1988-03-29 estimated the $5577 \AA$ line contribution to the $V$ band to lie between 0.10 and $0.29 \mathrm{mag}$. The $V / y$-band difference between our two sets of data (1978 vs. $1987 / 88)$ is in qualitative agreement with these estimates, and indicates that, due to the 5577 A line, the $y$ band data are at least $0.1 \mathrm{mag} / \square^{\prime \prime}$ brighter than the $V$ band ones. The difference in the $B$ band values is more surprising. There are no significant airglow or artificial lighting lines within our 3840/4160 A filter pass bands. Thus, the difference between the two periods is more probably due to a real sky brightness difference.

To compare our $B$ and $V$ sky brightness values with the previous measurements at other sites we list in Table 10 the representative minimum and maximum sky brightness for each set of observations. The minimum/maximum values given are averages of the three smallest/largest sky brightness values occuring in each set of data. The values refer to zenith. The corresponding solar $10.7-\mathrm{cm}$ flux value is also given.

We have done some measurements of the zenith distance dependence of the airglow at ESO and Calar Alto. We have found that a correction of $0.05 \mathrm{mag} / \square^{\prime \prime}$ is appropriate for $B$ and $V$ to correct the sky brightness from airmass 1.15 (a representative airmass for our nightly average values, see Fig. 1) to zenith. This correction corresponds, on average, to the results obtained by Walker (1970) for several sites.

The values given in Table 10 for ESO, La Silla are as follows: The $B$ and $V$ band given in bold numbers corresponds to the observed minimum and maximum sky brightness from the period February 5-8 1978 corrected by $0.05 \mathrm{mag}$ to zenith; the numbers following in parentheses are the averages of three minimum/maximum values out of the whole data set including the synthetic values. As can be seen from Table 10 the difference between the two $I_{B}(\mathrm{~min})$ values is minimal. The directly observed $I_{V}(\mathrm{~min})$ value is 0.11 mag brighter than the synthetic one. In this case we consider the synthetic value more appropriate because it represents an observing period (Dec. 1987) closer to the solar minimum. Because of the $5577 \AA$ line contribution this minimum value should probably still be corrected by at least $0.05-0.1 \mathrm{mag}$, as discussed above (we have, however, not applied this correction to the data presented in Table 10).

The actually observed $B$ band maximum brightness, $I_{B}(\max )$, is clearly not representative as it refers to a period of only average solar activity. The synthetic $I_{B}(\max )$ value should be adopted. For the $V$ band maximum 
Table 9. Comparison of intermediate band sky brightnesses (in $10^{-9} \mathrm{ergs} \mathrm{cm}^{-2} \mathrm{~s}^{-1} \mathrm{sterad}^{-1} \AA^{-1}$ ). For each value the lower limit is the average of the three lowest values and the upper limit is the average of the three highest values belonging to the data set (Haute Provence, Calar Alto and ESO). For Tsumeb the mean value with its standard error is given

\begin{tabular}{|c|c|c|c|c|c|c|c|}
\hline Site & \multicolumn{4}{|c|}{$I_{\lambda}$} & Dates & Solar min./max. date & Ref. \\
\hline \multirow{3}{*}{ Haute Provence } & $I(3670 \AA)$ & & $I(4400 \AA)$ & $I(5260 \AA)$ & & & \\
\hline & $254-491$ & & $187-341$ & $192-331$ & $1953-55$ & $1954.3(\min )$ & \\
\hline & $366-668$ & & $259-481$ & $210-412$ & $1957-59$ & $1957.9(\max )$ & 1. \\
\hline \multirow[b]{2}{*}{ Tsumeb (Namibia) } & & $I(3920 \AA)$ & $I(4900 \AA)$ & $I(5580 \AA)$ & & 1964.8(min) & \\
\hline & & $124 \pm 15$ & $236 \pm 14$ & $281 \pm 34$ & 1965-66 & $1968.9(\max )$ & 2. \\
\hline \multirow[b]{2}{*}{ Calar Alto } & $I(3500 \AA ̊)$ & $I(3920 \AA ̊)$ & $I(4670 \AA)$ & $I(5250 \AA)$ & & $1986.8(\min )$ & \\
\hline & $236-500$ & $170-322$ & $263-484$ & $263-481$ & 1989-93 & $1990.2(\max )$ & 3. \\
\hline \multirow[b]{2}{*}{$\mathrm{ESO}$} & $I(3500 \AA)$ & $I(3840 \AA)$ & $I(4670 \AA ̊ 丿)$ & $I(5467 \AA ̊)$ & & $1979.9(\max )$ & \\
\hline & $176-475$ & $153-329$ & $206-365$ & $261-746$ & 1979-88 & $1986.8(\min )$ & 4. \\
\hline
\end{tabular}

1. Barbier, 1956, 1961

2. Dachs, 1970

3. Leinert, et al., 1995

4. This paper

Table 10. Comparison of $B$ and $V$ zenith sky brightnesses at different sites. The minimum/maximum values given are averages of the three smallest/largest sky brightness values (nightly averages) given for each site. The solar $10.7 \mathrm{~cm}$ flux vlue is the average of the three nights in question

\begin{tabular}{|c|c|c|c|c|c|c|c|}
\hline Site & $I_{B}(\max )$ & $I_{B}(\min )$ & $I_{V}(\max )$ & $I_{V}(\min )$ & Solar flux & Corresponding dates & ref. \\
\hline $\mathrm{ESO}$ & $\begin{array}{r}\mathbf{2 2 . 7 4} \\
(22.20)\end{array}$ & $\begin{array}{r}\mathbf{2 2 . 9 7} \\
(22.94)\end{array}$ & $\begin{array}{r}\mathbf{2 1 . 6 9} \\
(20.85)\end{array}$ & $\begin{array}{r}\mathbf{2 1 . 9 1} \\
(22.02)\end{array}$ & $\begin{array}{r}164 \\
168 \\
161 \\
116 \\
162 \\
94\end{array}$ & $\begin{array}{l}\mathbf{7 8 - 0 2 - 0 5} \\
80-12-06 ; 80-06-08 ; 88-12-05 \\
\mathbf{7 8 - 0 2 - 0 8} \\
78-02-08 ; 87-12-16 ; 87-12-19 \\
\mathbf{7 8 - 0 2 - 0 7} \\
87-12-15 ; 87-12-16 ; 87-12-19\end{array}$ & 1. \\
\hline Calar Alto & $\mathbf{2 2 . 5 4}(22.30)$ & 23.05(22.98) & 21.16 & 21.83 & $\begin{array}{l}191 \\
176 \\
206 \\
156\end{array}$ & $\begin{array}{l}\mathbf{9 0 - 2 6 - 2 7} ; 89-05-03,04,05 \\
89-05-06 ; \mathbf{9 0 - 0 6 - 2 6} ; 93-06-21 \\
89-05-04 ; 91-06-11 ; 91-06-16 \\
90-06-23 ; 90-06-26 ; 91-06-16\end{array}$ & 2. \\
\hline San Benito Mt. & 22.37 & 23.08 & 21.32 & 22.07 & $\begin{array}{r}233 \\
78 \\
76\end{array}$ & $\begin{array}{l}80-04-11 ; 81-07-28 ; 82-06-22 \\
76-04-30 ; 87-04-25 ; 87-06-29 \\
76-04-30 ; 87-04-28 ; 87-06-29\end{array}$ & 3. \\
\hline Kitt Peak & 22.65 & 22.98 & 21.60 & 22.01 & $\begin{array}{r}114 \\
75 \\
114 \\
75\end{array}$ & $\begin{array}{l}88-01-21 ; 88-03-17 ; 88-06-14 \\
86-12-02 ; 86-12-30 ; 87-06-22 \\
87-11-20 ; 88-03-17 ; 88-06-14 \\
86-12-02 ; 86-12-31 ; 87-06-22\end{array}$ & 4. \\
\hline Hawaii & 22.27 & 23.03 & 21.21 & 22.05 & $\begin{array}{l}210 \\
142 \\
166 \\
102\end{array}$ & $\begin{array}{l}88-11-13 ; 89-03-28 ; 89-09-12 \\
87-08-26 ; 87-11-13 ; 89-06-10 \\
85-12-13 ; 88-11-13 ; 89-03-28 \\
86-06-02 ; 87-08-26 ; 88-07-18\end{array}$ & 6. \\
\hline $\begin{array}{l}\text { McDonald } \\
\text { Observatory }\end{array}$ & 22.54 & 23.01 & 21.54 & 21.92 & $\begin{array}{l}138 \\
156 \\
159\end{array}$ & $\begin{array}{l}60-02-04 ; 72-12-30 ; 73-01-12 \\
60-01-27 ; 72-01-11 ; 72-01-15 \\
60-01-27 ; 72-01-15 ; 73-01-08\end{array}$ & 7. \\
\hline
\end{tabular}

1. This paper

2. Leinert et al. (1995)

3. Walker (1988)

4. Pilachowski et al. (1989)

5. Lyutyi \& Sharov (1982)

6. Krisciunas (1990)

7. Kalinowski et al. (1975) 
brightness the same conclusion holds, except that here the synthetic $I_{V}(\max )$ is some $0.1-0.5 \mathrm{mag}$ too bright due to the $5577 \AA$ contribution.

Even without applying the correction factors discussed above (which would improve the La Silla minimum values) it can be concluded from Table 10 that the sky brightness at La Silla is very similar to the best among the other observing sites listed. We should note that our observing positions for the intermediate band photometry ("standard positions" in L134 and L1642) were located in the densest and darkest parts of the two dark clouds. At these positions there is still some excess surface brightness (scattered light) present above the surrounding empty sky level. This contribution amounts to $\sim 2-2510^{-9} \mathrm{ergs} \mathrm{cm}^{-2} \mathrm{~s}^{-1}$ sterad $^{-1} \AA^{-1}$ between 3500 - $5500 \AA$ (Mattila et al. 1996, in preparation). Subtracting these values from the directly observed sky brightness values would not make any noticeable change. It is also to be noted that in the other night sky measurements listed in Table 10 excess surface brightness components of similar magnitude were present, e.g. due to integrated starlight within the large measuring aperture.

\subsection{Spectral energy distribution of sky brightness}

We show in Fig. 7 the spectral energy distributions (SED) of the night sky brightness as observed at ESO. The average values for each observing period are presented as filled circles for the intermediate band data (dark clouds L134 and L1642) and as open circles for the uvgr data (clusters of galaxies). We show, for comparison, the spectral energy distribution for Mt. Palomar in the night 1972-11-28 as reported by Turnrose (1974). This observing date was $\sim 4$ years after the solar maximum of 1968.9. We do not have a corresponding year in the solar cycle; of our years, the year 1980 is at the highest on the solar cycle, and clearly gives larger sky brightnesses than Turnrose's data; 1987 is just past a minimum (8 years after maximum) and has a lower sky brightness than Turnrose; the best agreement is with our year 1983, which was 3.2 years after the maximum of 1979.9. This diagram also demonstrates the large differences in sky brightness between adjacent wavelength bands in the red part of the spectrum.

For comparison we show in Fig. 7 the SED for the zodiacal light at the two observed dark clouds, which are located at the ecliptic latitudes of $\beta \approx-36^{\circ}$ and $15^{\circ}$ for L1642 and L134, respectively. The galaxy cluster areas had similar ecliptic latitudes of between $\beta \approx-35^{\circ}$ to $-25^{\circ}$. These ZL spectra were obtained by scaling the ZL values $I_{U}, I_{B}$ and $I_{V}$ from Helios (Leinert et al. 1982) with the solar SED given by Labs \& Neckel (1970). As can be seen from the figure, the ZL contribution is always $\leq 50 \%$ of the total LONS.

\section{A new method for separation of zodiacal light and airglow}

\subsection{Description of the method}

Photometry of the zodiacal light has been performed during the past thirty years by several space-borne instruments, most notably by the Helios 1 and 2 solar probes (Leinert et al. 1982). Observing from outside the earth's atmosphere overcomes at once the problems caused by the tropospheric extinction and scattering as well as airglow. There are, however, other problems with the space-borne observations, such as the starlight subtraction (because of the large field of view) or limited spatial and time coverage, which ought to motivate at least some ground-based observational work in future.

So far the the most extensive ground-based zodiacal light photometry has been performed by Dumont and Sánchez (cf. Dumont 1976 and references therein). Because of the innovative and accurate techniques developed by Dumont for the separation of airglow and zodiacal light ("Method of Multiple Heights") this photometry has remained a standard source of reference in the era of spaceborne observations.

In this section we will present another method for the separation of zodiacal light and airglow. As in the method of Dumont, we utilize the correlated variations of the airglow in two adjacent bands. However, unlike in Dumont's method, we do not rely on the zenith distance dependence of the airglow but rather on the different spectral shapes of zodiacal light and airglow around $4000 \AA$. In our case both bands measure the airglow (quasi)continuum intensity, one just below and the other one just above the $4000 \AA$ discontinuity. The zodiacal light has a spectrum which is a direct copy of the solar Fraunhofer line spectrum in its narrow-band features (cf. Blackwell \& Ingham 1961a,b). We utilize here the discontinuity at $4000 \AA$ which is very prominent in the solar spectrum as well as in all late type stars. The intensity ratio for zodiacal light in our two filter bands was determined using the solar SED as published by Labs \& Neckel (1970), and we obtained for it the value $I_{\lambda}(3840 \AA) / I_{\lambda}(4160 \AA)=0.61$.

The ratio is distinctly different from the observed intensity ratio for the airglow. This fact enables us to achieve a separation of the two components by observing the same fixed position on the sky at different epochs with greatly differing, but correlated airglow contributions.

\subsection{Observations, reductions and results}

In Figs. 8 and 9 the night sky brightness, as observed in the filter band at $3840 \AA$, is displayed vs. the sky brightnesses in the the $4160 \AA$ band. Data from three separate observing periods, Dec. 1980, Dec. 1987, and Dec. 1988 are plotted in Fig. 8 for L1642. For the other target, L134 (Fig. 9), we had data for only one observing period in June 1979. It is apparent from these two figures that the sky 


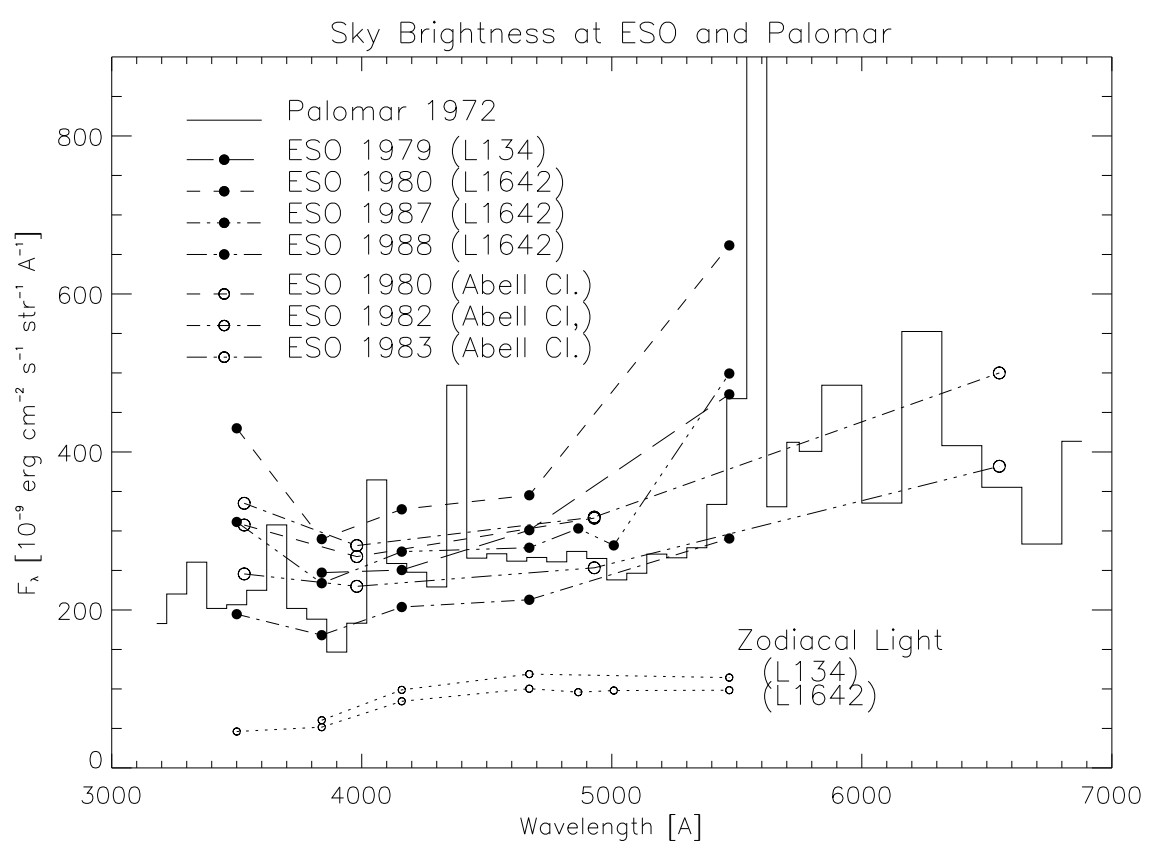

Fig. 7. Spectral energy distribution of the LONS at ESO. Solid dots refer to the intemediate-band data of dark clouds, open circles refer to the uvgr data of clusters of galaxies. For comparison, the ZL spectrum (relative form $=$ Solar spectrum) is shown for the two dark clouds, as well as the spectrum of the LONS on 1972-11-28 at the Palomar Observatory as reported by Turnrose (1974). Unit is $10^{-9} \mathrm{erg} \mathrm{cm}^{-2} \mathrm{~s}^{-1}$ sterad $^{-1} \AA^{-1}$

brightness variations in the two adjacent filter bands are tightly correlated. It can also be seen that the slope of the linear least-squares fit, shown as a continuous line, is close to unity for the data in Fig. 8 while it is somewhat steeper for the data in Fig. 9. It is apparent that the fitted line does not pass through the origin in either $\mathrm{c}$ ase. This is due to the presence of the zodiacal light. We have plotted in Figs. 8 and 9 the possible locations of zodiacal light data points corresponding to the intensity ratio 0.61 as a dashed line, labeled ZL. If only zodiacal light were present the observed values for any position on the sky should lie on this line. As can be seen from Figs. 8 and 9 the ZL line and the regression line fitted through the observations intersect at $I_{\lambda}(4160 \AA)=89$ and at 95 $10^{-9}$ ergs $\mathrm{cm}^{-2} \mathrm{~s}^{-1}$ sterad $^{-1} \AA^{-1}$ for L1642 and L134, respectively. This crossing points give the raw values for the zodiacal light intensity in these two directions. They have still to be corrected for atmospheric scattered light and extinction.

The observed night sky brightness as seen by a groundbased observer can be written in the general form:

$I_{\mathrm{obs}}=\left(I_{\mathrm{EBL}}+I_{\mathrm{ISL}}+I_{\mathrm{DGL}}+I_{\mathrm{ZL}}+I_{\mathrm{AGL}}\right) \mathrm{e}^{-\tau(z)}+I_{\mathrm{sca}}$, where the individual components refer to the extragalactic background light (EBL), integrated starlight (ISL), diffuse galactic light (DGL), zodiacal light (ZL), and airglow (AGL). All these extraterrestrial surface brightness components are subject to the tropospheric extinction by the factor $\mathrm{e}^{-\tau(z)}$, where the optical depth, $\tau(z)$, refers to the zenith distance of the observation. Part of the light which is removed by extinction from the above mentioned components is returned as scattered light, described by the term $I_{\text {sca }}$. The scattered light along a specific line of sight depends on the light distribution over the whole sky above the horizon. For a given fixed position (in $\alpha, \delta) \quad I_{\text {sca }}$ thus depends also on the time. Staude (1975) has performed extensive numerical calculations for the tropospheric scattered light with realistic surface brightness distributions for the ISL, ZL and AGL as the input light source. He has found that among the various approximative reduction techniques the recipe of Dumont (1965) gave the best agreement with the numerical results. We notice that in our case the components $I_{\mathrm{ISL}}$ and $I_{\mathrm{EBL}}$ are negligible, the former because of the selection of star-free observing areas. Nevertheless, the ISL still contributes to $I_{\text {sca }}$ by full weight and must be considered. In our case the "raw" ZL value, as obtained as the intersection point of the two lines shown in Figs. 8 and 9 is already free of airglow and also of airglow-induced scattered light (because the latter has the same intensity ratio $I(3840 \AA)$ / $I(4160 \AA)$ as the airglow itself).

For the further reduction steps we apply the recipies of Dumont (1965): (1) we subtract the tropospheric Rayleigh scattered light due to ZL and ISL; (2) we correct the intensity thus obtained for Rayleigh extinction ; (3) the aerosol scattering is strongly forward-directed and is assumed to cancel against extinction for extended surface brightness sources. We end up with the extraterrestrial surface brightness values of $I_{0}(4160 \AA)=84.4$ and 88.7 


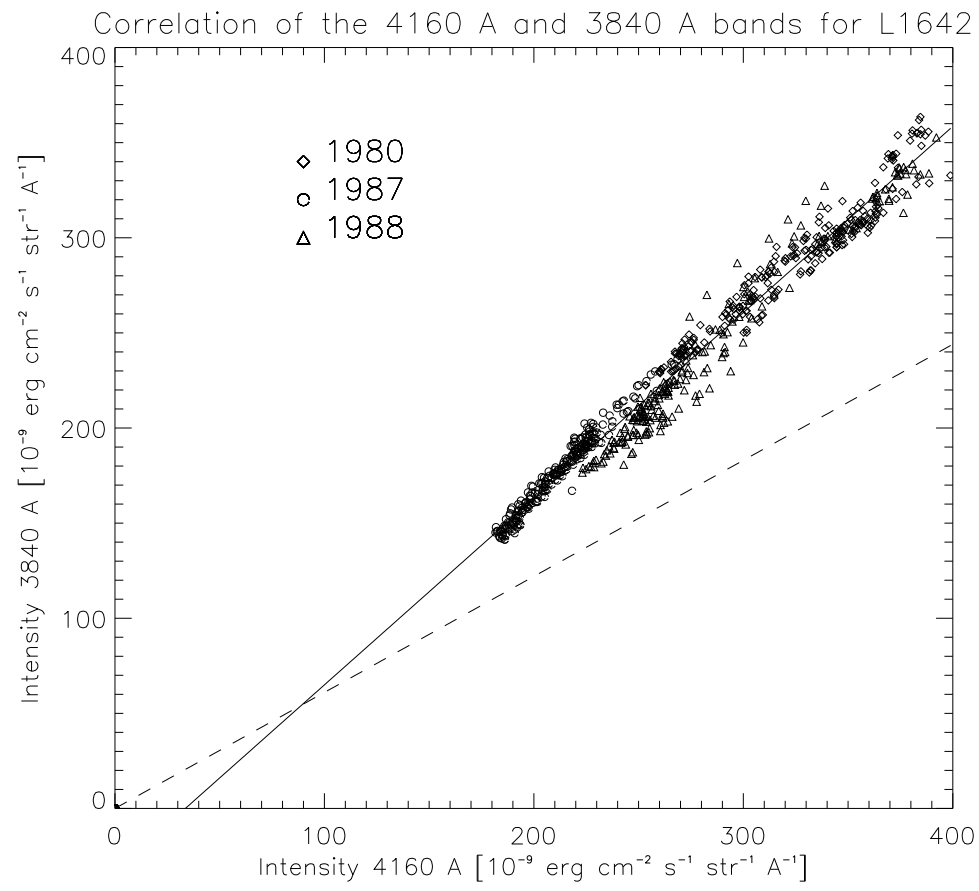

Fig. 8. Correlation of night sky brightnesses at 3840 and $4160 \AA$ as observed during different nights in the direction of L1642. The unit is $10^{-9}$ erg $\mathrm{cm}^{-2} \mathrm{~s}^{-1}$ sterad $^{-1} \AA^{-1}$

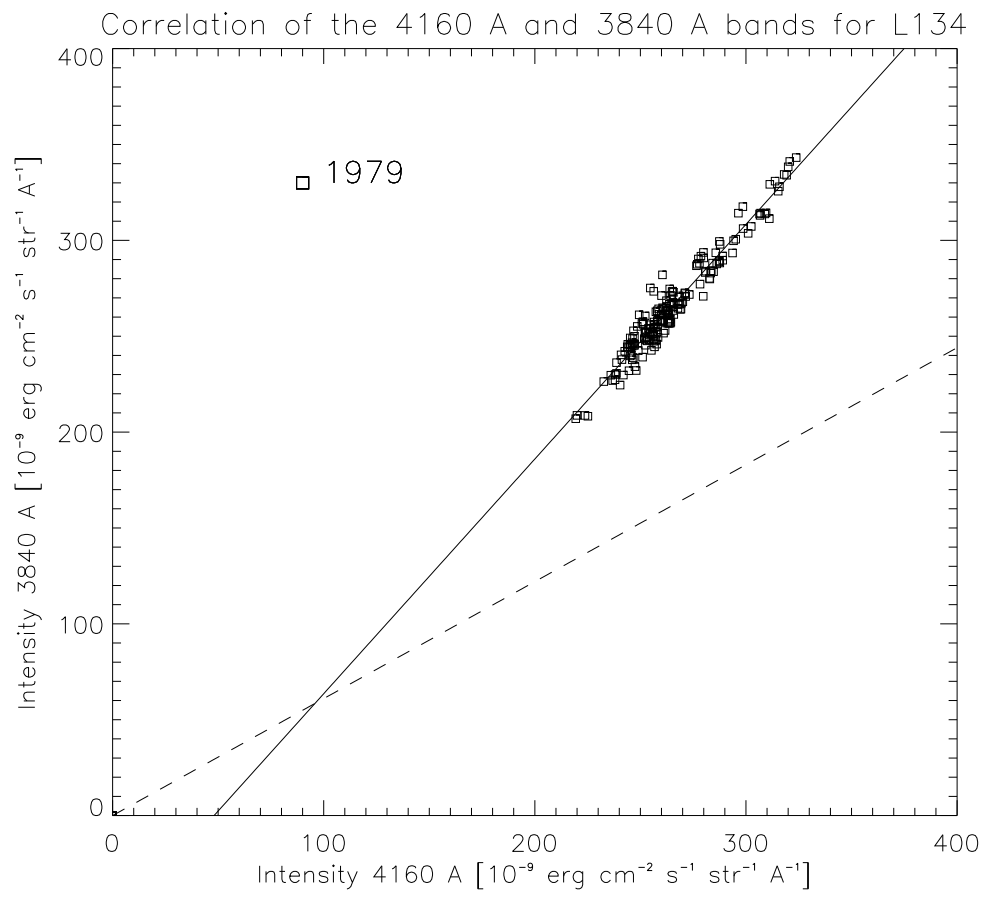

Fig. 9. Correlation of night sky brightnesses at 3840 and $4160 \AA$ as observed during different nights in the direction of L134. The unit is $10^{-9} \mathrm{erg} \mathrm{cm}^{-2} \mathrm{~s}^{-1}$ sterad $^{-1} \AA^{-1}$ 
$10^{-9} \mathrm{ergs} \mathrm{cm}^{-2} \mathrm{~s}^{-1}$ sterad $^{-1} \AA^{-1}$ for L1642 and L134, respectively. As a final step we have to subtract the diffuse galactic (scattered) light contributions from our dark cloud targets. They were accurately determined by comparing the dark cloud position with several OFF positions outside the nebula which are free of dust to very low levels (see Mattila et al. 1991; Laureijs et al. 1987). The DGL value at $4160 \AA$ at the observed positions was 13.5 and $510^{-9} \mathrm{ergs} \mathrm{cm}^{-2} \mathrm{~s}^{-1}$ sterad $^{-1} \AA^{-1}$ in L1642 and L134, respectively. Thus our final values for the ZL at $4160 \AA$ at these two positions are:

L1642: $I_{\mathrm{ZL}}=7110^{-9} \mathrm{ergs} \mathrm{cm}^{-2} \mathrm{~s}^{-1} \mathrm{sterad}^{-1} \AA^{-1}$ L134: $I_{\mathrm{ZL}}=8410^{-9} \mathrm{ergs} \mathrm{cm}^{-2} \mathrm{~s}^{-1}$ sterad $^{-1} \AA^{-1}$.

We can compare these values with the results of Helios 1 and 2 (Leinert et al 1982). The Helios photometry was performed at $\beta \approx \pm 16^{\circ}$ and $\pm 31^{\circ}$, i.e. very close to $\beta(\mathrm{L} 1642)=-35.9^{\circ}$ and $\beta(\mathrm{L} 134)=15.3^{\circ}$. After small corrections for the ZL gradients according to LeveasseurRegourd \& Dumont(1980) we end up with the following Helios values at $4160 \AA$ :

L1642: $I_{\mathrm{ZL}}=86 \pm 1510^{-9} \mathrm{ergs} \mathrm{cm}^{-2} \mathrm{~s}^{-1} \mathrm{sterad}^{-1} \AA^{-1}$ $\mathrm{L} 134: I_{\mathrm{ZL}}=102 \pm 15 \quad 10^{-9} \mathrm{ergs} \mathrm{cm}^{-2} \mathrm{~s}^{-1} \mathrm{sterad}^{-1} \AA^{-1}$. Since our values also have an uncertainty of at least \pm 15 $10^{-9} \mathrm{ergs} \mathrm{cm}^{-2} \mathrm{~s}^{-1}$ sterad $^{-1} \AA^{-1}$, we can conclude that the agreement with Helios is excellent. Although this good agreement may be fortuitous, we can still offer a possible explanation for the difference between Helios and our results: We have subtracted the diffuse galactic (scattered) light contribution (cirrus), a component which - thanks to IRAS - is now known to be present almost all over the sky, but has not been considered by the ZL observers so far.

For any further application of the method the following remarks should be made:

(1) Although the correlation between the two filters just below and above the $4000 \AA$ discontinuity appears to be a very tight one, it is not certain that the extrapolated least-squares line really passes through the zero point (i.e. $\left.I_{\mathrm{AGL}}=0\right)$; in other words, there may be a constant offset in either one of the pass bands. This will especially be the case if even a slight amount of pollution through artificial lighting is present. The strong Hg line at $4047 \AA$ may be very disturbing if included in the upper filter pass band. A high mountain site is preferrable in order to reduce the effects of atmospheric scattered light and extinction.

(2) Filters as close as possible to the $4000 \AA$ discontinuity should be used in order to avoid differences in atmospheric extinction and scattered light.

\section{Summary and conclusions}

We have performed intermediate and broad band photometry of the night sky brightness during $1978-1988$ at the European Southern Observatory with an observing technique that allows elimination of starlight and permits ac- curate comparison of measurements from different nights and years. Our main conclusions are the following:

1. More than $50 \%$ of the continuum sky brightness at ESO during moonless nights is due to airglow. The airglow intensity varies strongly both during individual nights, from night to night, and from year to year. We do not find any regular patterns in the nightly variations.

2. The night sky brightness variations in the different intermediate band continuum filters between 3500 and $5470 \AA$ are well correlated. This is in agreement with the "covariance group I" found by Barbier (1956). However, variations in the broad band $B V R I$ filters as observed in 1978 are poorly correlated.

3 . The long-term variations of the night sky brightness in the airglow "pseudocontinuum" between 3500 and 6500 $\AA$ are correlated with the solar activity. For the nine-year period covered by our intermediate band observations we have found a change of sky brightness by a factor of 1.5 in the blue spectral range, in good agreement with the earlier findings of Walker (1988). This solar-activity-dependent long-term variability must be taken into account when studying the long-term increase of light pollution effects, or when comparing measurements from different sites obtained at different dates.

4. We have compared our intermediate band sky brightness measurements with similar data from Haute Provence, Tsumeb (Namibia) and Calar Alto. We have found that the La Silla sky brightnesses are similar or lower than the three other sites.

We have compared our sky brightness measurements at La Silla with the broad band $B$ and $V$ values available in the literature for other good observing sites. We have found that the La Silla sky brightness is as good as that of the very darkest sites, e.g. San Benito Mountain or Hawaii.

These results are contrary to the public rumours that, possibly due to the South Atlantic Anomaly, the sky in Chile would be brighter than e.g. in South Africa or southern parts of North America.

5 . Based on the good correlation of the airglow variations in the two adjacent bands at 3840 and $4160 \AA$ we have suggested a new method for the separation of the airglow and zodiacal light.

\section{References}

Barbier D., 1961, Contr. de l'Inst. d'Astroph. Paris Ser. B 216, 35

Barbier D., 1956, The Airglow and the Aurorae, Special Suppl. 5 to the J. Atm. Terr. Physics, p. 38

Bessel M.S., 1990, PASP 102, 1181

Blackwell D.E., Ingham M.F., 1961a, MNRAS 122, 113

Blackwell D.E., Ingham M.F., 1961a, MNRAS 122, 129

Chamberlain 1961, Physics of the Aurorae and Airglow. London, Academic Press, p. 516

Christophe-Glaume J., 1965, Ann. de Geoph. 21, 1

Dachs J., 1968, Beiträge zur Physik der Atmosphäre 41, 76

Dachs J., 1970, A\&A 6, 155 
Dumont R., 1965, Ann. Ap. 28, 265

Dumont R., 1976, Ground-based observations of the zodiacal light. In: Elsässer H., Fechtig H. (eds.) Interplanetary Dust and Zodiacal Light, Proc. IAU Coll. No 31. Springer-Verlag, p. 85

Garstang R.H., 1989, Ann. Rev. A\&A 27, 19

Garstang R.H., 1986, PASP 98, 364

Hayes D.S., 1985, in: Hayes D.S., Pasinetti L.E., Davis Philip A.G. (eds.) Calibration of Fundamental Stellar Quantities. Reidel, Dordrecht, p. 225

Hecht J.H., Ramsay Howat S.K., Waltersheid R.L., Isler J.R., 1995, J. Geophys. Res. (in press)

Iriarte B., Johnson H., Mitchell R., Wisniewsky W., 1965, Sky \& Telesc. 30, 21

Johnson H., 1955, Ann. Ap. 18, 292

Kalinowski K.J., Roosen R.G., Brandt J.C., 1975, PASP 87, 869

Kent S.M., 1985, PASP 97, 165

Krisciunas K., 1990, PASP 102, 1052

Labs D., Neckel H., 1970, Sol. Phys. 15, 79

Laureijs R., Mattila K., Schnur G., 1987, A\&A 184, 269

Leinert Ch., Richter I., Pitz E., Hanner M., 1982, A\&A 110, 355

Leinert Ch., Väisänen P., Mattila K., Lehtinen K., 1995, A\&AS 112,99

Levasseur-Regourd A.C., Dumont R., 1980, A\&A 84, 277

Lyutui V.M., Sharov A.S., 1982, AZh 59, 174

Massey P., Gronwall, Pilachowski, 1990, PASP 102, 1046

Massey P., Straabel K., Barnes J., Anderson E., 1988, ApJ 328, 315

Mattila K., Leinert Ch., Schnur G., 1991, in: RoccaVolmerange B., Deharveng J.M., Tran Thanh Van J. (eds.)
The Early Observable Universe from Diffuse Backgrounds. Editions Frontieres, p. 133

McNally D., 1994, The Vanishing Universe, Adverse Environmental Impacts on Astronomy. Cambridge University Press

Neckel Th., Chini R., 1980, A\&AS 39, 411

Oke J.B., 1974, ApJS 27, 21

Oke J.B., Gunn J.E., 1983, ApJ 266, 713

Pilachowski C., Africano J., Goodrich B., Binkert W., 1989, PASP 101, 707

Rayleigh IV, Lord (R.J.Strutt), 1928 Proc. Roy. Soc. London A 119,11

Rayleigh IV, Lord (R.J.Strutt), Jones H.S., 1935, Proc. Roy. Soc. London A 151, 22

Roach F., 1955, Ann. Geophys. 11, 100

Rosenberg N., Zimmerman S.P., 1967, Planet. Space Sci. 15, 863

Schnur G., Mattila K., 1979, Mitt. Astron. Ges. 45, 196

Schnur G., 1980, Photoelectric surface photometry of extended sources. In: Crane P., Kjär K. (eds.) Proc. ESO workshop on two dimensional photometry, European Southern Observatory, p. 365

Schwarz H.E., Melnick J., 1989, The ESO Users Manual 1990, European Southern Observatory

Staude J., 1975, A\&A 39, 325

Stone R.P.S., 1977, ApJ 218, 767

Thuan T.X., Gunn J.E., 1976, PASP 88, 543

Turnrose B.E., 1974, PASP 86, 512

van Rhijn P.J., 1921, Publ. Astr. Lab. Groningen 31, 1

Walker M.F., 1988, PASP 100, 496

Walker M.F., 1977, PASP 89, 405

Walker M.F., 1970, PASP 82, 674 